Missed Diagnosis

\title{
Basosquamous carcinoma, a wolf in sheep's clothing? Report of 3 cases.
}

\author{
B.F. Johnson, P.J. Moore*, J.R. Goepel and D.N. Slater \\ Royal Hallamshire Hospital \& Weston Park Hospital, Sheffield and Rotherham District General Hospital, \\ Rotherham, UK.
}

\begin{abstract}
Summary: Basosquamous carcinoma of the skin is a lesion with specific histological features and recognized metastatic potential. Three cases of metastatic basosquamous carcinoma are reported, two of which were rapidly fatal. They illustrate that diagnostic features may be absent in a small biopsy and that metastasis is usually associated with a poor prognosis.
\end{abstract}

\section{Introduction}

Cutaneous basal cell carcinoma (BCC) and squamous carcinoma (SCC) display characteristic histology and behaviour allowing appropriate management. However, a small number of tumours with intermediate histological features are recognized, termed metatypical or basosquamous carcinoma (BSC). They illustrate that the histology is clearly defined, but characteristic features may not be apparent in a small biopsy. In addition, these tumours have a definite metastatic potential which needs to be recognized. Three illustrative cases of basosquamous carcinoma with metastases are presented and the management discussed.

\section{Case reports}

\section{Case 1}

A 58 year old male had a $1.5 \mathrm{~cm}$ diameter lesion removed from his anterior chest wall. Histology showed an adequately excised BCC. Lymphatic invasion was noted, but the risk of metastasis reported to be extremely unlikely. Two years later, a local recurrence was irradiated (5500 cGy in 14 doses) and after only a partial response, widely excised and skin grafted. Histology confirmed recurrent BCC 'com-

Correspondence: B.F. Johnson, F.R.C.S., Royal Hallamshire Hospital, Glossop Road, Sheffield, S10 2JF, UK.

*Present address; Scunthorpe General Hospital, Scunthorpe, South Humberside, UK.

Accepted: 10 April 1989 pletely excised'. He was discharged from follow-up 4 months later.

After 5 more years he returned with a painful, fixed® left axillary mass. Surgical excision proved impossibje and biopsy only was performed. Metastatic BSC watsoo confirmed and radiotherapy ( $5000 \mathrm{cGy}$ in 20 dose). administered. He remains well, without furthero disease, after 4 more years.

Case 2

A 68 year old female had a pedunculated lesion $\vec{\circ}$ removed from the upper outer aspect of her left thigh. $\exists$ Histology showed incompletely excised BSC.

Four years later, she presented with enlarged left inguinal lymph nodes which were surgically excised eno bloc with the overlying skin. Histology of the nodes 3 . revealed metastatic BSC, but the resection margins were free of tumour.

After 4 more months she returned with lym-ฮ̊ phoedema of her left leg and biopsy of indurated groin skin revealed recurrent carcinoma. Following a short응 course of palliative radiotherapy to her groin and $>$ pelvis, she died in a hospice 6 weeks later.

Case 3

A 63 year old debilitated man had a 5 year history of a slowly growing lesion on his chest. It was a $9 \mathrm{~cm} \times 110$ $\mathrm{cm}$ ulcer fixed to the manubrium and upper body of sternum. Biopsy suggested a BCC and he died of bronchopneumonia before local radiotherapy was completed. Post-mortem revealed extensive bone deposits of BSC. 


\section{Discussion}

Many malignant cutaneous tumours are easily classified as either classic BCC or SCC with correct prediction of their biological behaviour. Some tumours, however, are intermediate in their histology, while others are somewhat variable from one microscopic field to another: these groups of tumours are classified as metatypical or basosquamous carcinomas. ${ }^{1}$

Although some authors dispute the existence of BSC, ${ }^{2}$ Borel gives a very clear description of their histology. ${ }^{3}$ In brief, there are infiltrating nests of basaloid cells with relatively uniform nuclei that palisade at the periphery. However, within individual nests there are larger, more eosinophilic squamous cells that may show intercellular bridges of keratinization. The first biopsy may be purely BCC while a recurrence is BSC. They need to be distinguished from keratinizing BCC which shows abrupt keratinization in some cell nests without intervening squamous cells, and ulcerating BCC which may show squamous metaplasia towards the exposed surface. Cases reported as $\mathrm{BCC}$ with metastases often show features of basosquamous carcinoma. ${ }^{4}$

Our cases 1 and 3 both illustrate the problems of sampling a large lesion. Tumour heterogeneity may explain why the histology showed BCC areas without basosquamous features, with consequent inaccurate prediction of their behaviour. Case 1 may represent a change in the character of the tumour with time. Previous reports have suggested that the clinical behaviour resembles SCC with a recurrence rate of $12 \%$ to $51 \%$ in the head and neck. ${ }^{3,5}$ Our three cases emphasize the risk of recurrence and metastasis. BSC is most frequent on the head and neck ${ }^{3-5}$ and like most

\section{References}

1. Ten Seldam, R.E.J. \& Helwig, E.B. Histological Typing of Skin Tumours. World Health Organisation, Geneva, 1974, p. 50 .

2. Lever, W.F. \& Schaumburg-Lever, G. Histopathology of the Skin, 6th edition. Lippincott, Philadelphia, 1983.

3. Borel, D.M. Cutaneous basosquamous carcinoma. Review of the literature and report of 35 cases. Arch Pathol 1973, 95: 293-297.

4. Farmer, E.R. \& Helwig, E.B. Metastatic basal cell carcinoma: a clinicopathologic study of seventeen cases. Cancer 1980, 46: 748-757. actinic cancers has a low incidence of metastasis. One group $^{6}$ has calculated a $5 \%$ metastatic rate over a 20 year period. Another group found a mean latent period of 10 years for the development of metastases from the time of diagnosis of the primary, but this varied from 0 to 30 years. ${ }^{4}$ Our cases all arose from unexposed surfaces and this may be an adverse prognostic factor. Metastatic BSC is difficult to treat and associated with a poor prognosis. Farmer and Helwig ${ }^{4}$ found the average life expectancy was only 1.6 years from the time of detection of metastases.

Of possible treatment modalities, surgical excision of the primary skin BSC was associated with the least number of recurrences, ${ }^{4}$ although the importance of adeqate excision is exemplified by our case 2 . Radiotherapy or cryotherapy can also give acceptable results when the primary lesion is unsuitable or the patient unfit for surgery.

Careful follow-up is a 'counsel of perfection' in view of the variable latent interval which is often 10 years or more. However, if a patient who has had a BCC previously excised presents with metastasis, this should prompt a review of the histology before assuming there is a second primary.

In conclusion, BSC may be difficult to diagnose, but its enhanced malignant potential should be recog nized. When the primary lesion shows diagnostio histology, adequate surgical excision should be advised.

\section{Acknowledgement}

We thank Mr C.H. Talbot, M.Chir., F.R.C.S., Consultant Surgeon at the Royal Hallamshire Hospital, for allowing us to study cases 1 and 2.
5. Schuller, D.E., Berg, J.W., Sherman, G. \& Krause, C.J. Cutaneous basosquamous carcinoma of the head and neck: a comparative analysis. Otolaryngol Head Neck Surg 1979, 87: 420-427.

6. Lopez de Faria, J. \& Nunes, P.H.F. Basosquamous cell carcinoma of the skin with metastases. Histopathology 1988, 12: 85-94. 